\title{
The prodromal phase of first episode mania: The patients and caregivers perspective
}

\author{
Eline Regeer ${ }^{1}$, Eva Maassen ${ }^{1}$, Lotte Maathuis², Barbara Regeer², Ralph Kupka1,3 \\ ${ }^{1}$ Altrecht Institute for Mental Health Care, Outpatient clinic for bipolar disorders, Utrecht, The Netherlands. \\ ${ }^{2}$ VU University Amsterdam, Athena Institute, Faculty of Earth and Life Sciences, Amsterdam, The Netherlands. \\ ${ }^{3}$ Amsterdam University Medical Center, Department of Psychiatry, Amsterdam, The Netherlands.
}

\section{Background:}

To decrease the negative consequences related to bipolar disorder, a timely diagnosis, followed by an adequate treatment is necessary. However, there is an average 10-year delay in diagnosing bipolar disorder ${ }^{1}$, hampering the application of effective therapeutic interventions. The aim of this study was to improve early recognition of bipolar disorder by exploring the prodromal phase of recently diagnosed patients with bipolar I disorder and their caregivers. To elaborate and gain new perspectives on prodromal symptoms revealed by quantitative research $^{2-5}$ a qualitative research method was chosen ${ }^{6}$.

\section{Objectives:}

Clarify the prodromal phase of the first episode mania from the perspective of patients and the caregivers by a qualitative research method.

\section{Methods:}

Semi-structured interviews were conducted with 15 patients with bipolar I disorder, who had their first manic episode within the last 5 years and their caregivers. These interviews were developed to elicit information about early symptoms e.g. behavioral, psychological and physical changes before a first episode mania. Thematic and open coding were used to analyze the data.

\section{Results:}

Half of the patients mentioned a family member who suffers from bipolar disorder. Almost all patients experienced a depressed mood earlier in life. Within the year prior to the first manic episode patients and caregivers mentioned psychological changes (willing to achieve, mood lability), behavioral changes (increased involvement in activities, being absorbed in activities, talkativeness) and physical changes (alternating energy levels, restless, sleeplessness, turned biorhythm). In most cases, the changes followed after a major life event. Caregivers additionally mentioned that the normal behavior and personality characteristics of the patient became more pronounced.

\section{Conclusions:}

A prodromal phase before a first mania is recognized by patients and caregivers. Caregivers could name more precise the behavioral, psychological and physical changes in the prodromal phase.

\section{I can be very happy and be gloomy, I recognise this from an early age (patient, male, age of 58)}

Yes, that you became a caricature of yourself, the things characterising a person were magnified (caregiver, female , friend)

\section{Yes, that restlessness expresses itself in.. keep doing things. We already talk fast, but then even faster (caregiver, mother)}

I am reliving the last few months before the mania. She can't take a distance from... She can't let things go (caregiver, male, partner)

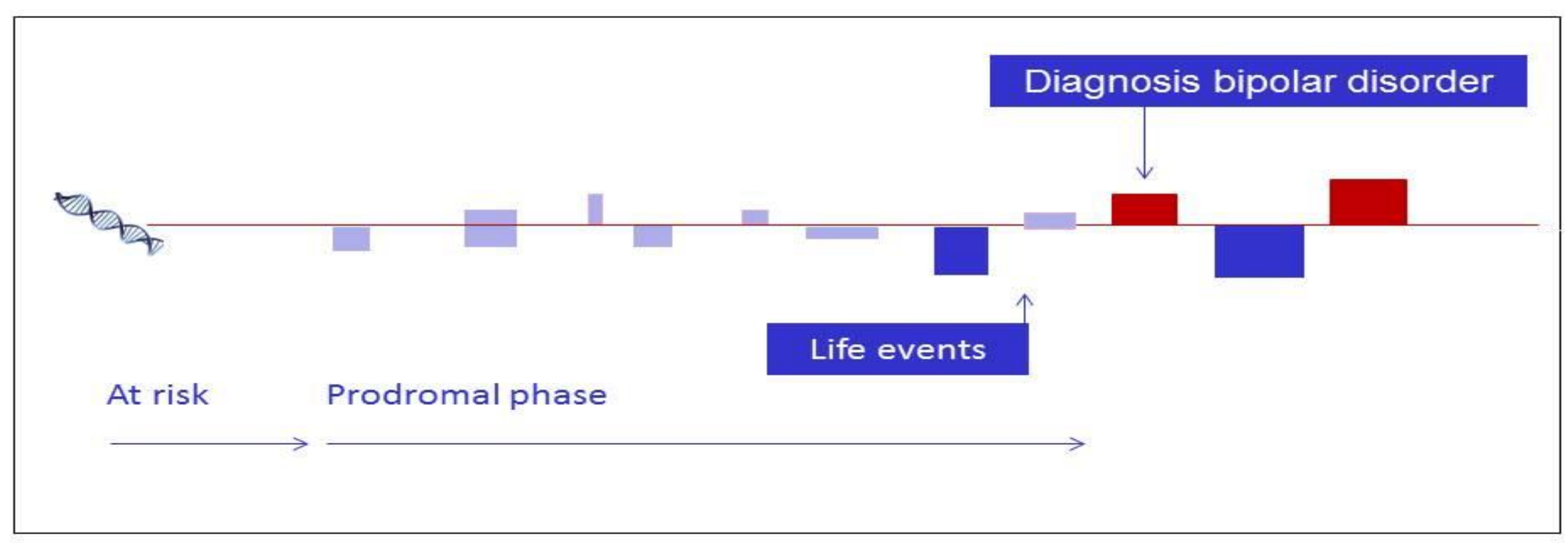

The things he said were plausible, thougth it was odd, but I did not think something was wrong, (caregiver, mother)

Figure 1. Timeline of the development of bipolar disorder

${ }^{1}$ Drancourt et al., 2013, 2Correl et al., 2014,

${ }^{3}$ Feadda et al .,2015, ${ }^{4}$ Van Meter et al., 2016

${ }^{5}$ Pfenning et al., 2017, ${ }^{6}$ Benti et al., 2014

For more information contact Eline Regeer: e.regeer@altrecht.nl

I see links others do not see, in that way I am very creative, I used to create beautiful things (patient, female, age of 24) 